\title{
Electronic Resource Management ${ }^{1}$
}

\section{Problemstellung}

Seit Mitte der 1990er Jahre nehmen elektronische Ressourcen im Angebot der Bibliotheken eine immer wichtiger werdende Rolle ein. Anfangs waren es Datenbanken und bibliographische Nachweissysteme wie etwa CAS, die in lokalen Netzwerken der Universitäten einen komfortablen Weg zum Nachschlagen von Informationen und Fakten anboten. Mit Einführung des HTTP-Protokolls entstand eine sehr einfache Möglichkeit, Datenbankinformationen für den Nutzer intuitiv erfassbar zu präsentieren. Neben einer Umstellung von lokalen Datenbanken zu webbasierten waren vor allem die Katalogsysteme plötzlich mit den OPACs präsent; gleichzeitig lösten die elektronischen die gedruckten Bibliographien ab. Besonders dramatisch entwickelte sich aber der Sektor der Zeitschriften. Waren es um 1998 einige tausend Zeitschriften vornehmlich aus dem STM-Bereich, die elektronisch zugänglich waren, sind zehn Jahre später in der Central Knowledge Base (CKB) von SFX (Ex Libris) 65000 Zeitschriften elektronisch nachgewiesen, so dass gesagt werden kann, dass praktisch keine wissenschaftliche Zeitschrift mehr ohne eine elektronische Parallelausgabe existiert. Inzwischen sind auch neue Bücher häufig parallel in gedruckter und elektronischer Form zu erhalten. Darüber hinaus wird durch zahlreiche Digitalisierungsprojekte eine größer werdende Zahl von älteren Büchern online im Netz angeboten.

Die wachsende Bedeutung der elektronischen Medien lässt sich unter anderem an der Entwicklung von Erwerbungsetats ablesen. So gibt das zweischichtige Bibliothekssystem der FU Berlin inzwischen über ein Drittel der Erwerbungsmittel für elektronische Ressourcen aus. Damit verbunden ergibt sich in der Regel die Herausforderung, die elektronischen Ressourcen bei gleich bleibender oder gar abnehmender Personalkapazität adäquat zu verwalten.

Im Jahre 2001 hat Timothy D. Jewell in einem vielbeachteten Report ${ }^{2}$ erstmals auf einige der wesentlichen Probleme bei der Bearbeitung von elektroni-

1 Der vorliegende Beitrag enthält Elemente des Beitrags von Kowalak, Mario: Electronic Resource Management. In: Handbuch Bestandsmanagement in Öffentlichen Bibliotheken. Hrsg. von Frauke Schade u. Konrad Umlauf. Berlin: De Gruyter (2012) (Bibliotheks- und Informationspraxis 46). S. 375-390.

2 Vgl. Jewell, Timothy D.: Selection and Presentation of Commercially Available Electronic Resources. Issues and Practices. Washington, DC: Digital Library Federation 2001. http://www.clir. org/pubs/abstract//reports/pub99 (14.8.2012). 
schen Ressourcen in Bibliotheken hingewiesen. Ausgehend davon wurde durch die Digital Library Federation eine Arbeitsgruppe gebildet, die 2004 ihren Abschlussbericht ${ }^{3}$ vorlegte. Mit diesem Report wurde eine auch von Produzenten von Bibliothekssystemen mitentwickelte Übersicht der administrativen Daten für die Bearbeitung elektronischer Ressourcen sowie funktionale Anforderungen an solche Systeme aufgestellt. Ebenso werden in diesem Papier erstmals typische Workflow-Szenarien der Bearbeitung elektronischer Ressourcen beschrieben.

Für die einzelne Bibliothek stellt sich das Problem, wie die elektronischen Produkte in Geschäftsgänge eingebunden werden. Dabei müssen alle verwaltungstechnischen Aspekte wie Rechnungsabläufe, revisionssichere Inventarisierung und bibliothekarische Erschließung umgesetzt werden. Gleichzeitig ist eine optimale Präsentation und Vermittlung der Ressourcen an die Nutzer notwendig. Dies soll mit der Nebenbedingung einer möglichst integrierten und effizienten Bearbeitung ohne Doppelaufwand erfolgen. Bibliotheksverbünde und Konsortien können die Bibliotheken - gerade bei konsortial bereitgestellten Produkten - bei der Verwaltung elektronischer Ressourcen unterstützen, stellen aber, zum Beispiel bei auftretenden Zugriffsproblemen, nicht die vorrangigen Ansprechpartner der Bibliotheksnutzer dar. So kommt es in der Praxis teilweise zu zersplitterter oder auch doppelter Datenpflege, die letztlich in unterschiedlichen Bedürfnissen und Voraussetzungen der einzelnen Bibliotheken auf der einen und der Verbünde und Konsortien auf der anderen Seite begründet ist.

In den seit Mitte der 90er Jahren in den Bibliotheken eingesetzten integrierten Bibliothekssystemen ist die Verwaltung elektronischer Medien bisher nicht vorgesehen. Natürlich können und werden mit einigen Workarounds elektronische Medien in den Katalogsystemen bearbeitet, aber es sind und bleiben Fremdkörper in der Bearbeitungsroutine.

Bei den elektronischen Ressourcen verbieten sich traditionelle Methoden der Katalogisierung weitgehend. Zum einen kann die Bibliothek in der Regel nur sehr begrenzt unmittelbar über ihr elektronisches Angebot verfügen, da dieses in den meisten Fällen online auf entfernten Servern der Anbieter bereitgestellt wird. Jede Änderung auf diesen Servern kann eine Änderung des referenzierenden URL im eigenen Katalog nach sich ziehen. Zum anderen ist die Lizenzierung insbesondere bei den Zeitschriftenpaketen so dynamisch, dass die Bestandsänderungen in den großen Paketen nur mit großem personellen Aufwand und mit zeitlicher Verzögerung eingearbeitet werden können.

3 Vgl. Jewell, Timothy et al.: Electronic Resource Management. Report of the DLF ERM Initiative. Washington, D.C.: Digital Library Federation 2004. http://www.diglib.org/pubs/dlf102/ (14.8.2012). 
Aber auch viele wichtige Informationen, die mit der Vertragsbearbeitung und -verwaltung verbunden sind, werden im Katalogsystem nicht abgebildet und müssen anderweitig dokumentiert werden. Hierzu wurden, basierend auf den Vorarbeiten der erwähnten DLF-ERM-Initiative, in den letzten Jahren einige Softwareanwendungen entwickelt, die mehr oder weniger gut die komplexe Vertragsverwaltung erledigen.

Ein nur unzureichend bearbeitetes Problem stellen die Konsortialbeschaffungen dar. Aus Sicht einer einzelnen Bibliothek bedeuten Konsortialbeschaffungen, dass die unterschiedlichen Konsortialführer für die Bibliothek wie ein sonstiger Lieferant zu behandeln sind. Von dort kommen ggf. Rechnungen, müssen Titellisten und Nutzungsstatistiken angefordert werden. Dorthin werden auch Reklamationen gerichtet.

Ein weiterer Problemkomplex stellt die Paketerwerbung dar. Hier gibt es teilweise unterschiedliche Angebote für elektronische Bücher (E-Books) und elektronische Zeitschriften (E-Journals). Die Paketerwerbung bei Zeitschriften leitet sich häufig von laufenden Subskriptionen für gedruckte Journals eines Verlages ab, E-Book-Pakete werden ohne Orientierung an konventionellen Bibliotheksbeständen sowohl von Verlagen als auch von Aggregatoren angeboten, gemeinsam ist allen Angeboten, dass man keinen Einfluss auf die Paketzusammensetzung hat, die überdies nicht selten innerhalb der Laufzeit geändert wird. Diese Änderungen lassen sich lokal nur schwer in den Nachweissystemen aktuell und korrekt abbilden.

Dass elektronische Ressourcen zuweilen nur unzureichend erschlossen und in den Nachweisinstrumenten der Bibliothek daher nicht immer vollständig und umfassend suchbar sind, hat neben der Mengenproblematik auch mit der zum Teil mangelhaften Qualität der von Anbieterseite bereitgestellten Metadaten zu tun. Vielfach werden die ausgelieferten Metadaten vor dem Import in lokale Nachweissysteme daher durch eine aufwendige Normalisierungsprozedur geschickt und streckenweise auch noch einzeln nachbearbeitet, etwa im Bereich der Sacherschließung. Gerade mit modernen Nachweismitteln wie DiscoverySystemen entstehen dann beim Retrieval ernsthafte Verluste, da unzureichend erschlossene Medien beim „Drill Down“ nicht mehr gefunden werden können.

Bei gleich bleibender oder sinkender finanzieller Ausstattung der Bibliotheken werden verstärkt Statistiken über die Nutzung elektronischer Medien als Instrument der Bestandsentwicklung eingesetzt. Nachdem mit COUNTER ein gemeinsamer Standard definiert wurde, ist es mit Werkzeugen wie „Ustat“ mit vertretbarem Aufwand möglich, die Statistiken unterschiedlicher Quellen, 
Anbieter und Plattformen vergleichend zu betrachten und Schlüsse für die Präsentation, das Marketing und die Beschaffung von E-Ressourcen zu ziehen. ${ }^{4}$

Wir möchten uns im Folgenden auf die klassischen elektronischen Medien wie bibliographische Datenbanken, E-Journals und E-Books beschränken. Das weite Feld der frei zugänglichen Informationsquellen soll dagegen weitgehend ausgespart werden. Auch spezielle Medien wie Ton-, Bild- und Videodokumente, die häufig noch speziellere Bearbeitungsschritte erfordern, sollen hier nicht betrachtet werden. Ebenfalls nicht ausführlicher gewürdigt werden können Volltextdatenbanken und -sammlungen, die in den Geschäftsgängen aus Personalkapazitätsgründen vielfach nur auf Ebene der Hauptaufnahme, nicht jedoch auf Ebene der enthaltenen Einzeltitel erschlossen und damit den Nutzern nicht in gebotenem Maße präsentiert werden.

\section{Lebenszyklen elektronischer Ressourcen und Potentiale von ERM-Systemen}

Geschäftsgänge für konventionelle und elektronische Ressourcen unterscheiden sich grundlegend. Neue Aufgaben und Prozessschritte wie die Unterstützung der Lizenz- und Vertragsverhandlungen, das Management von Accounts und Zugriffsberechtigungen, Überblick über laufende Trials, die Überwachung und Dokumentation von Störungsfällen oder die Aggregation und Auswertung von Nutzungsstatistiken, um nur einige Beispiele zu nennen, treten im Zusammenhang mit elektronischen Ressourcen neu hinzu und sind mit dem konventionellen Instrumentarium des Bibliotheksmanagements, mit Hilfe von integrierten Bibliothekssystemen, nicht adäquat zu bewältigen. Die folgende Grafik

\footnotetext{
4 Aus den Statistiken der Webserver können recht genaue Informationen über das Nutzungsverhalten und die Zahl der Downloads erhalten werden. Allerdings sind diese Statistiken den Bibliotheken in der Regel nicht unmittelbar zugänglich und die von den Verlagen angebotenen Daten unterscheiden sich in Qualität, Aussagekraft und Präsentationsform erheblich. Hier ist mit den COUNTER-Formaten inzwischen ein Standard etabliert worden. Zum einen wird die Art definiert, wie die Nutzung gezählt wird. Damit ist erstmalig eine Vergleichbarkeit der Statistiken zwischen den COUNTER-fähigen Ressourcen möglich. Zum anderen ist eine einheitliche Präsentation der Daten definiert worden. Dies ist die Voraussetzung dafür, dass diese Daten in Werkzeugen wie „Ustat“ geladen und vergleichend untersucht werden können. Allerdings ist dies angesichts der Vielzahl von Anbietern, mit denen eine große Bibliothek arbeitet, immer noch ein mühseliges und zeitaufwendiges Verfahren. Einen großen Fortschritt stellt daher SUSHI dar, mit dessen Hilfe Programme wie „Ustat“ in der Lage sind, die Daten automatisch abzuholen und einzulesen. Weiteres unter http://www.projectcounter.org/about.html (14.8.2012).
} 
beschreibt schematisch wesentliche Unterschiede in den Geschäftsgängen für konventionelle und elektronische Ressourcen.
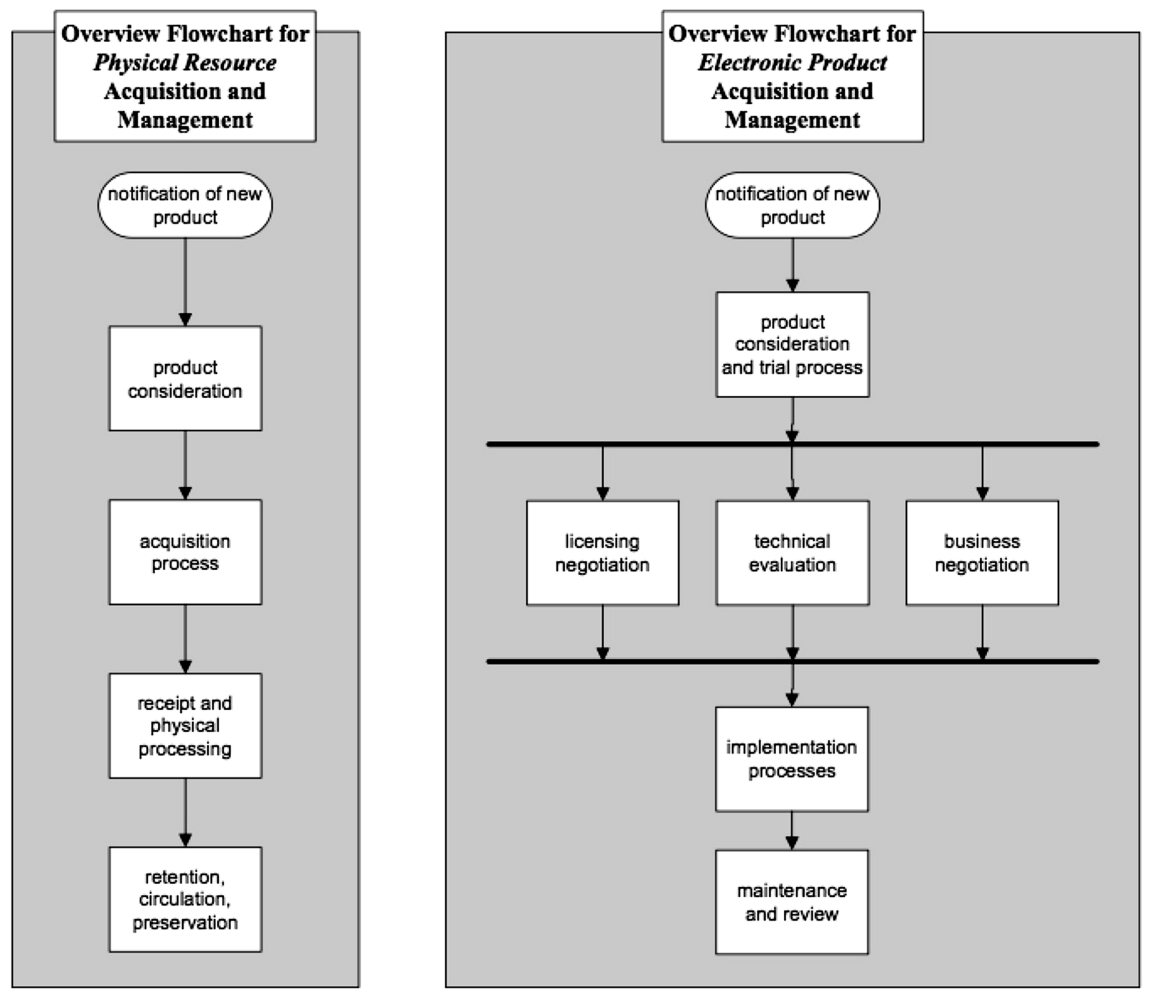

Abb. 1: Unterschiede in Geschäftsgängen für konventionelle und elektronische Ressourcen. Quelle: Electronic Resource Management of the DLF ERM Initiative.

Es wird schnell deutlich, dass die Bereitstellung elektronischer Ressourcen eine Vielzahl komplexer Arbeitsabläufe im Hintergrund benötigt, die zumeist zyklisch wiederholt werden müssen. In der Fachliteratur ist diese besondere Dynamik elektronischer Medien, die sich beispielsweise in der häufigen Änderung von Zugangsadressen und -modalitäten, einer gewaltigen Bandbreite von Geschäftsmodellen oder regelmäßig überarbeiteten Suchoberflächen äußert, vielfach in Analogie zur Betrachtung lebender Organismen gebracht worden. So kann von „lebendigen“ Medien gesprochen werden ${ }^{5}$, die in zyklisch verlaufenden Bearbei-

5 Vgl. zuletzt u.a. bei Collins, Maria u. Jill E. Grogg: Building a Better ERMS. In: Library Journal (2011) H. 4. S. 22-28. 
tungsroutinen („Life Cycle“) finanziert, freigeschaltet, bereitgestellt, überwacht, evaluiert werden.

Bereits ab 2004 wurden, basierend auf dem Framework der Arbeiten der DLFERM-Initiative, kommerzielle und Open Source-Produkte entwickelt und auf den Markt gebracht, welche die komplexen Vorgänge und Aufgaben im Rahmen des Lebenszyklus‘ elektronischer Ressourcen dokumentieren, strukturieren und operationalisieren sollen.

Verschiedentlich werden ERM-Systeme mit einem integrierten bzw. eigenem Workflow Management eingesetzt. Angesichts der Koexistenz und des Einsatzes zahlreicher externer Systeme und Werkzeuge (Tools) ist es in der Regel jedoch sehr schwer, die ERM-internen Workflows mit den Geschäftsgängen der Bibliothek und den weiteren Systemen zu einem arbeitsfähigen Ganzen zu verbinden.

Betrachten wir im Folgenden einen vereinfachten und schematisierten Prozessverlauf („Life Cycle“) für lizenzierte elektronische Ressourcen. Welche Managementaufgaben können von professionellen ERM-Systemen übernommen werden? Was sind deren spezifische Potentiale und Einsatzgebiete?

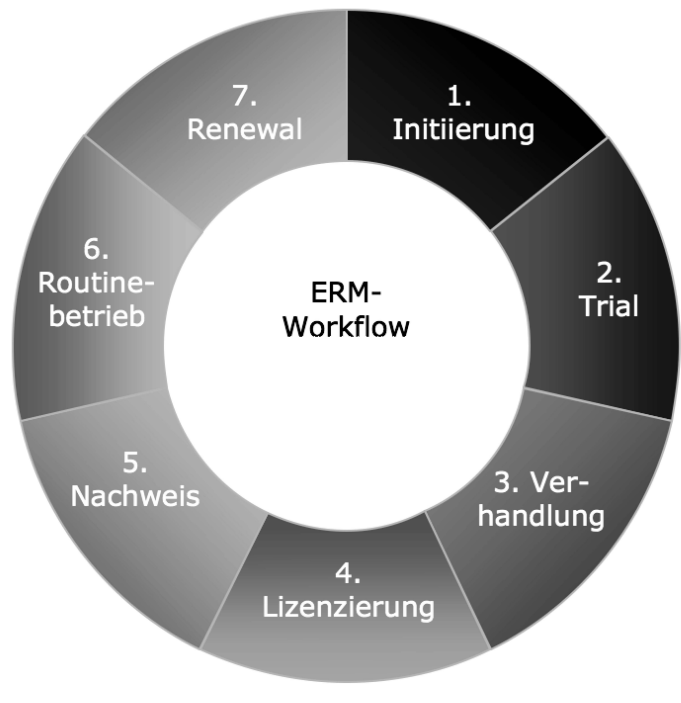

Abb. 2: „Life Cycle“ für lizenzierte elektronische Ressourcen, eigene Darstellung.

\section{Phase: Initiierung}

Nachdem die Bibliothek auf eine potentiell zu erwerbende elektronische Ressource aufmerksam geworden ist, kann mit Hilfe eines professionellen ERM-Sys- 
tems eine Art Marktsichtung probiert werden. Ausschlaggebend hierfür ist die Bereitstellung und die Qualität einer so genannten „Knowledge Base“, die das Angebot und den Markt für E-Ressourcen möglichst genau abbilden soll. Mittels einer „Overlap Analysis“ kann nachvollzogen werden, ob die fragliche Quelle in unterschiedlichen Aggregatorangeboten oder bei unterschiedlichen Plattformen zugänglich ist.

\section{Phase: Trial}

Wie viele und welche Tests hat die Bibliothek gegenwärtig laufen? Lohnen sich weitere Tests? Aus dem ERM-System heraus können Tests für E-Ressourcen in angeschlossenen Nachweissystemen als solche gekennzeichnet werden. Ebenso können zu beteiligende Mitarbeiterinnen und Mitarbeiter über den Test gezielt informiert und in die Lage versetzt werden, ihre Bewertungen der Testquelle strukturiert im ERM-System abzulegen. Automatisierte Alerting Services können das Ablaufen der Testphase gezielt kommunizieren.

\section{Phase: Verhandlung}

Indem das ERM-System über seine Knowledge Base verschiedene, auch vergleichende, Produktinformationen vorhält, unterstützt es gegebenenfalls die Verhandlungsposition der Bibliothek. Hilfreich ist nicht zuletzt die Übersicht, welche weiteren elektronischen Ressourcen beim selben Anbieter bereits erworben wurden oder laufend bezogen werden.

\section{Phase: Lizenzierung}

Hier liegt ein zentrales Potential professioneller ERM-Systeme. Die gewöhnlich hochkomplexen Vertragstexte können analytisch abgebildet werden, so dass essentielle Aussagen zu Rechten und Pflichten schnell auffindbar sind. In einzelnen ERM-Systemen gibt es allein für diesen Bereich über 100 Erschließungsfelder. Ausgewählte Vertragspassagen können aus dem ERM-System heraus gezielt in angeschlossene Nachweissysteme ausgegeben werden. Die Verträge können eingescannt, mit OCR-Software bearbeitet und dann retrievalfähig in das ERMSystem hochgeladen werden. 


\section{Phase: Bestandsnachweis}

Aus dem ERM-System lassen sich Metadaten in verschiedener Form in Nachweissysteme (DBIS, EZB, lokaler OPAC) exportieren. Dies ist allerdings mit erheblichem Aufwand verbunden. Die Knowledge Base des ERM-Systems muss außerdem laufend mit der Knowledge Base des eingesetzten Link Resolvers synchronisiert werden.

Insbesondere bei Buch- und Zeitschriftenpaketen muss $\mathrm{zu}$ jedem Vertrag eine Liste der Objekte, also der Zeitschriften oder Bücher, die zu diesem Vertrag gehören, gepflegt und in die Nachweissysteme weitergegeben werden. Diese Verknüpfung zwischen Vertrag und den einzelnen Titeln ist in traditionellen Bibliotheksverwaltungssystemen nicht möglich und einer der Gründe für die Entwicklung eigenständiger ERM-Systeme.

\section{Phase: Routinebetrieb}

Anders als konventionelle Ressourcen, die nach Erwerb und Erschließung an ihre Bestimmungsorte wandern und in der Regel keine weitere Bearbeitung mehr erfordern, verursachen elektronische Medien im Routinebetrieb zahlreiche Folgearbeiten. Zugangs- und Kontaktdaten müssen möglichst zentral abgelegt und für alle in die Bearbeitung elektronischer Ressourcen involvierten Mitarbeiterinnen und Mitarbeiter zugänglich sein. Nutzungsstatistiken müssen regelmäßig erstellt werden; sie bilden die Basis für spätere Kosten-Nutzen-Analysen, die ebenfalls in professionellen ERM-Systemen vorgesehen sind. ERM-Systeme dienen darüber hinaus als Dokumentationshilfen bei der regelmäßigen Erfassung von aufgetretenen Zugriffsunterbrechungen oder Beschwerden von Nutzern. Erst wenn diese Vorgänge konsequent erfasst sind, können Nutzungsprobleme gezielt und systematisch angegangen werden. Gegenüber dem Anbieter der betreffenden elektronischen Ressource kann bei Verhandlungen dann besser argumentiert werden.

\section{Phase: Renewal}

Von besonderer Bedeutung ist hier eine Alert-Funktion, die die Bibliothek rechtzeitig vor Ablauf eines Vertragszeitraums an die anstehende Lizenzerneuerung oder Kündigung erinnert. Dazu gehören weitere ebenfalls im ERM verankerte Funktionen wie das Evaluieren anhand der Kosten-Nutzen-Analyse oder die erneute Vergleichsanalyse oder Marktsichtung, die dabei helfen, eine fundierte Entscheidung vor dem Auslaufen des Lizenzvertrags zu treffen. 


\section{Electronic Resource Management im weiteren Sinn}

Wie an der zuvor dargelegten Beschreibung der Potentiale von ERM-Systemen deutlich geworden ist, stellen DLF-ERM-basierte Systeme der ersten Generation wichtige Funktionalitäten im Backend-Bereich zur Verwaltung elektronischer Ressourcen in Bibliotheken bereit. Die seinerzeit verbreitete Annahme, ERMSysteme könnten sämtliche Aspekte der Erwerbung, Erschließung und Bereitstellung von E-Ressourcen in Bibliotheken bewerkstelligen, hat sich in der Praxis schnell als Fehleinschätzung erwiesen. ERM-Systeme der ersten Generation sind vielmehr umfangreiche Arsenale von Werkzeugen (Tools), die erst im sinnvollen Zusammenspiel ein leistungsfähiges Management von elektronischen Zeitschriften, Büchern und Datenbanken in Bibliotheken ermöglichen.

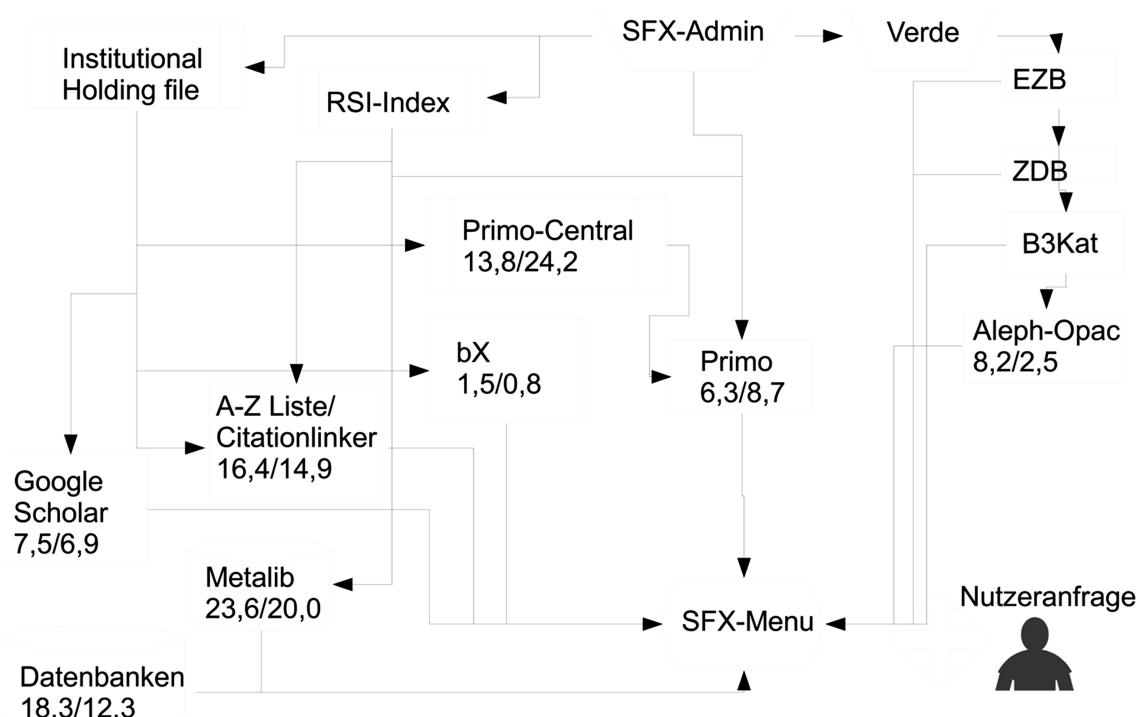

Abb. 3: Bestandinformation: Von VERDE/SFX über die Endnutzersysteme zum SFX-Menu. Die Zahlenwerte (in Prozent) beziehen sich auf die Herkunft der Anfragen an das SFX-System, stichprobenartig untersucht jeweils im Februar 2011 und 2012.

Insbesondere die Bereitstellung des ständig wechselnden elektronischen Bestands ist eine Herausforderung für die Datenpflege. Hier eignen sich der traditionelle Katalog und dessen auf Einzeltitel bezogene Bearbeitung nur sehr schlecht. Die Bestände von E-Journals werden im deutschsprachigen Raum in der EZB nachgewiesen. Dabei reduziert sich die Arbeit für eine Bibliothek auf den lokal lizenzierten Bestand, da Pakete und Nationallizenzen zentral gepflegt 
werden. Für den Nutzer ist die EZB jedoch weniger praktisch, da in jedem Einzelfall nicht nur gesucht werden muss, ob eine Zeitschrift auch elektronisch zugänglich ist, sondern auch, weil aus den Bestandsangaben nicht unmittelbar herausgelesen werden kann, ob die gesuchte Ausgabe verfügbar ist. Einfacher ist dies für den Nutzer, wenn es ein gepflegtes Link Resolver-System in der Bibliothek gibt.

Am Beispiel des kombinierten Einsatzes eines kommerziellen ERM-Systems, eines kommerziellen Link Resolvers sowie eines weiteren Suchwerkzeugs an der FU Berlin soll verdeutlicht werden, wie hier den Nutzern jeweils die aktuelle Information präsentiert wird.

In Abb. 3 zeigen wir am Beispiel der Arbeitsumgebung in der FU Berlin die Konzentration der Verwaltung elektronischer Ressourcen in VERDE. Durch die Konzentration der Pflege der Verträge in VERDE und der damit verknüpften Pflege der Bestandsdaten in SFX ist die jeweils aktuelle Information über elektronische Zeitschriften und Bücher immer verfügbar. Lediglich für die Erschließung der Datenbanken ist SFX nicht zu verwenden, hierfür steht das Präsentationsund Suchwerkzeug MetaLib zur Verfügung, das auch die verteilte Suche in mehreren Datenbanken unterstützt.

Jeder in MetaLib angezeigte Treffer bzw. die Treffer in den meisten Datenbanken verfügen über Links auf unser SFX-System, womit der direkte Zugriff auf die gewünschten Artikel im Volltext ermöglicht wird.

Suchmaschinen und Werkzeuge wie die A-Z-Liste, Primo oder auch Google Scholar verwenden die beiden Schnittstellen RSI-Index und Google-Holding-File bzw. Primo-Export File, um die Verfügbarkeit der Ressourcen zu bestimmen. Im Erfolgsfall wird eine OpenURL in das SFX-System importiert. Durch die asynchrone Bereitstellung der Daten, in der Regel nachts, im Fall von Google einmal pro Woche, werden gelegentlich falsche Verfügbarkeiten dargestellt, die aber durch den SFX-Link abgefangen werden. Dieses Vorgehen ist nicht auf E-Journals beschränkt, es wird analog auf andere E-Medien wie E-Books angewendet. Eine besondere Bedeutung kommt dem neuen Dienst Primo Central ${ }^{6} \mathrm{zu}$. Durch diesen Dienst wird die Wahrnehmung und Nutzung der elektronischen Ressourcen spürbar gesteigert. ${ }^{7}$

Der traditionelle Zeitschriftennachweis im lokalen Katalog ist aufwändig. Ein automatisches Übertragen der Zeitschrifteninformation aus VERDE in die EZB ist

6 Es handelt sich bei Primo Central um einen von Ex Libris angebotenen Metadaten-Index mit mehreren hundert Mio. Datensätzen, der bibliographische Daten und Volltext-Indexate von Verlagen, Aggregatoren und Agenturen enthält.

7 Die Zunahme, gemessen an Zugriffen, die über den Link Resolver SFX generiert werden, beträgt in der FU Berlin ca. $15 \%$. 
wegen der komplett unterschiedlichen Datenbankstruktur nicht mit vertretbarem Aufwand möglich. Derzeit erfolgt dieser Transfer für die ca. 5000 lokalen E-Journals an der FU Berlin manuell. Die Übertragung der Zeitschriftendaten aus der EZB über die bestehenden Schnittstellen in ZDB und B3Kat in den Aleph-OPAC ist eine eingespielte Routine. Da mittlerweile durch den direkten Export aus der Knowledge Base nach Primo der Nachweis der elektronischen Ressourcen für unsere Nutzer gegeben ist, wird sich die aufwendige Prozedur des lokalen Zeitschriftennachweises spätestens mit der Einstellung des Aleph-OPACs erübrigen.

Aus den SFX-Statistiken lässt sich auch die Herkunft einer OpenURL bestimmen. Die in Abb. 3 dargestellte prozentuale Verteilung für die FU Berlin stammt aus Stichproben im Februar 2011 und im Februar 2012 und zeigt, dass Suchmaschinen und Metadaten-Indices wie Primo Central für unsere Nutzer immer wichtiger werden und dass die Bedeutung des Nachweises von elektronischen Ressourcen in den traditionellen Katalogen dagegen abnimmt.

\section{Ausblick}

Mit der wachsenden Bedeutung von elektronischen Ressourcen für Bibliotheken ändert sich deren Selbstverständnis grundlegend. Diese Veränderungen sind häufig als Paradigmenwechsel - „from Holding to Access“, von der Hol- zur Bringbibliothek - beschrieben worden. Musste ein Nutzer, um ein gedrucktes Buch zu bekommen, früher zwangsläufig die Bibliothek aufsuchen, so ist es heute auf der Grundlage elektronischer Angebote leicht möglich, auf Inhalte zuzugreifen, ohne die Bibliothek zu betreten. Hier geht dann auch schnell das Bewusstsein dafür verloren, dass hinter der Bereitstellung elektronischer Inhalte zentrale bibliothekarische Dienstleistungen stecken. Denn den meisten Nutzern, die sich über ihr Hochschulnetz im Internet bewegen, bleibt verborgen, dass sie über Suchmaschinen auf kostenpflichtige Inhalte zugreifen, die ihnen nur aufgrund der Lizenzierung durch ihre Hochschulbibliothek zugänglich sind.

Auch die Präsentations- und Nachweissysteme der Bibliotheken müssen in diesem Zusammenhang auf den Prüfstand gestellt werden. Nach Untersuchungen der Autoren gehen ca. 90 \% der Downloads aus etablierten Fachzeitschriften nicht auf die klassischen bibliothekarischen Nachweissysteme zurück, sondern stammen offensichtlich aus externen Quellen wie Suchmaschinen oder individuellen Bookmarksammlungen. Neben einem verbesserten Branding, das die Herkunft der Bereitstellung für lizenzierte Inhalte deutlicher herausstellt, müssen die Attraktivität und das Leistungsvermögen bibliothekarischer Präsentationsund Nachweissysteme dringend gesteigert werden. Mit der Einführung von inte- 
grierten suchmaschinenbasierten Discovery-Systems, „One Stop Shops“, unternehmen Bibliotheken in den letzten zwei, drei Jahren Schritte in diese Richtung.

Die Bearbeitung und Bereitstellung von Medien und Informationsquellen in Bibliotheken befindet sich derzeit in einem enormen Umbruch. Mit Alma, Sierra und Worldshare stehen neue Produkte in den Startlöchern, die versprechen, zumindest einen Teil der bisherigen Probleme auf der Grundlage des „Unified Resource Management“ im integrierten Back Office zu beheben. Gemeinsam ist allen Produkten, dass es sich um $\mathrm{SaaS}^{8}$-Angebote handelt, also nicht mehr um lokal installierte Systeme. Vielmehr sind die Systeme in der Cloud und greifen auf eine gemeinsame Datenstruktur zu, so wie wir dies bisher aus den Verbundkatalogen kennen. Electronic Resource Management ist hierbei integraler Bestandteil der Datenbearbeitung und erfolgt nicht mehr getrennt von den gedruckten Werken. Hiermit wird es sehr viel leichter, die nutzerfreundliche Verbindung zwischen gedruckten und elektronischen Ausgaben bei Zeitschriften und Büchern herzustellen.

8 Software as a Service. 